\title{
SIMULATION AND MODELING OF ANN- BASED PROGNOSIS TOOL FOR A TYPICAL AIRCRAFT FUEL SYSTEM HEALTH MANAGEMENT
}

\author{
Vijaylakshmi S. Jigajinni $^{1}$ and Vanam Upendranath ${ }^{2}$ \\ ${ }^{1}$ Department of Electronics and Communication Engg., Basaveshwar \\ Engineering College, Bagalkot-587 102, Karnataka, India \\ ${ }^{2}$ Aerospace Electronics and Systems Division, CSIR-National Aerospace \\ Laboratories, Bengaluru-560017, Karnataka, India
}

\begin{abstract}
The ability to predict the aircraft fuel system health/operating condition and possible complications that occur during the long flight of an aircraft helps to improve the performance of the aircraft engine. Prognostics and Health Management (PHM) methodology includes fault detection, diagnosis, and prognosis. In this paper, we propose an Artificial Neural Network (ANN) based fault prognosis tool for a typical aircraft fuel system. Prognostics method using ANN's promise to provide a new approach to manage the fuel flow and fuel consumption of aircraft engine more effectively. This method identifies the presence of faults and mitigates them to maintain a proper fuel flow to the engine. Overlooking the presence of any faults in time could potentially be catastrophic which can lead to possible loss of lives and the aircraft as well. The developed tool works on the logical rules developed as per the engine's fuel consumption and quantity of fuel flow from the tanks. Here, we discuss the algorithm and the results of using ANN models to predict the health condition of the fuel system of aircraft.
\end{abstract}

\section{KEYWORDS}

Aircraft Fuel System, ANN, Fault Analysis, Diagnosis, Prognosis, HEalth Management

\section{INTRODUCTION}

Prognostics and Health Management (PHM) is the study of breakdown mechanisms and lifecycle management of a system [1]. It is a method that helps to assess the consistency of a system under its operating conditions to analyze the time of failure and mitigate the system risks [2]. An aircraft is a complex system of system operating as a group of interrelated systems [3]. Every aircraft system is responsible for safe operation.

Prognostics is the process of prediction based on present and prior conditions. Diagnostics pertains to the recognition and separation of faults or failures $[4,5]$. The goal of prognostics is to assess the overall future healthiness or condition of a system. It also deals with the prediction of the quality of a system including the Remaining Useful Life (RUL) of the system. In an aircraft, fuel to the engine is made to flow through fuel pipelines. A malfunction in any of the components, like, leakage in tanks, pump breakdown, pipeline leakage, and stuck valve etc., may lead to improper functioning of the fuel system as well and can result in the failure of the mission.

Natarajan Meghanathan et al. (Eds) : SAI, ICAITA, CSITA, ISPR, Signal - 2018

pp. 83-93, 2018. (C) CS \& IT-CSCP 2018

DOI : $10.5121 /$ csit.2018.81008 
In this work, a simulation model is developed to monitor and manage the health condition with a rule-based prognostics mechanism thus helping to make such predictions possible. The process of prognostics is a mathematical computation mechanism that predicts the future health of a complex system, fuel system in this context, based on the amount of past and current data available. The ultimate predictions made are based on data collected from multiple tanks with warnings, alerts, and safety measures. Continuous availability of useful data facilitates in improving the ability to diagnose and predict the effective functional life of a system. As the complexity of a given system increases, it also makes identification, isolation and finding the root cause of a fault in the system very difficult [6], thus increasing the work of the maintenance engineers. With these increasing demands on the safety of systems and dependability, a broad range of fault detection, diagnostic and prognostic methodologies have been projected in the literature [7].

Artificial Intelligence (AI) techniques based on neural networks are effective for modeling the complete health management of aeroplane fuel system. An ANN model can imitate a non-linear relationship between the required input and predicted output with good precision [8]. ANN is trained properly before it is used to model as per our required input-output relationship of the fuel system. Automatic updates of ANN model consider the data for any changes in working conditions of the considered system [9]. This study focuses on proper management of the flow of fuel to the engine by isolating the faults and mitigating them using this ANN prognosis tool.

\section{ARTIFICIAL NEURAL NETWORK (ANN)}

The main aim of the proposed prognostic model is to build a feed-forward mechanism using Artificial Neural Networks, to regulate input parameters to obtain the desired results. Learning and training process of the input-output patterns of the fuel system is done by a rule-based mechanism. This method helps to learn and adapt not only from environmental changes but also from changes in the output i.e. fuel consumption by the engine.

Different types of sensors are installed in an aircraft system. As sensors become smaller and smarter, the use of such sensors helps to gather a large volume of data which can be processed for prognostics [10]. Artificial Neural Networks models match with the biological neural systems that process parallel information [11]. ANN consists of two layers connected to the peripherals: an input layer to collect the data and an output layer to represent the result of the network. An example of a simple neural network is as shown in Figure 1. $X_{1}, \ldots, X_{n}$, represents the 'n' number of input signals and $W k l, \ldots, W k n$, represents the weights associated with each signal. These weighted inputs are added in a summing junction and an output $Y k$ is obtained through the activation function $F$.

In this neural network model, the summation function aggregates a weighted sum of inputs and the activation function converts the sum into the final output of the network [12]. Among the different training methods, Back Propagation(BP) is the most efficient one. Learning in the neural network is achieved by collecting the information in the form of training the data set. The weights are considered based on the type of training algorithm adopted. 


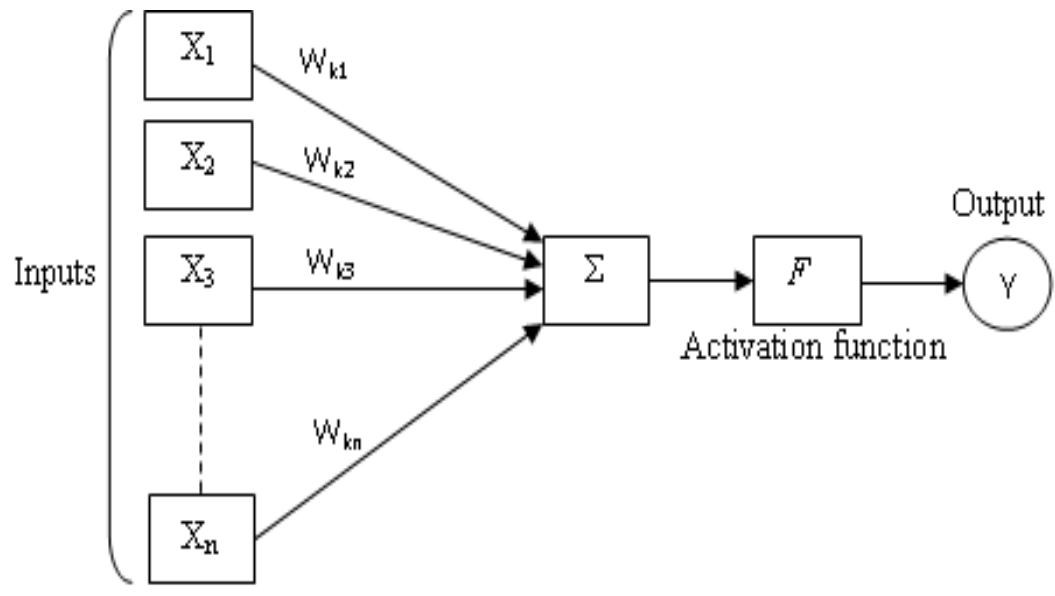

Figure1. A Neural Network model

This prognostic model includes four layers; an input layer, two intermediate hidden layers and an output layer of neurons. The feed-forward neural network equations for each step are as shown:

$$
\begin{gathered}
\mathrm{V}_{\mathrm{k}}=\sum_{j=1}^{n} W k j X j \\
Y^{\prime}(k)=S\left(V_{k}\right) \\
Y=\theta\left(\sum_{k=1}^{n} Y^{\prime}(k)\right)
\end{gathered}
$$

Proper training of the neural network model once done can be used for any type of incomplete or new data. The response obtained give predictions based on the inputs and adjusted weights accordingly. The prognostics engine uses input data (the fuel flow rate) and historical information (previous engine consumption rate) to train the ANN model for making predictions in relation to a working condition. The output function is described as:

$$
V_{k}=f\left(W_{k 1}, W_{k 2}, \ldots, W_{k n}\right)
$$

The model with the least error level was considered by comparing results by training the model with a different number of layers with multiple iterations.

\section{Simulation of THE PROPOSEd PROgNOSIS TOOL}

Figure 2 shows the block diagram of the prognosis tool with aircraft fuel tanks, pumps and pipeline routes. Generally, the fuel tanks in the aircraft are in the aircraft's fuselage and wings [13]. A typical small aircraft fuel system model is simulated in the Simulink, by considering eight centrifugal fuel pumps. Out of eight fuel pumps, two pumps are used for fuel delivery between the left and right wings and two other pumps for backup for any emergency conditions and remaining four main pumps for fuel delivery to the engines.

The primary objective of this work is to monitor continuously the fuel flow to the engines without any restrictions, to reach the required fuel consumption rate. Any fault occurred in the fuel tanks is detected and mitigated by the ANN-based controller. In a fuel system, there are various parameters which change due to change in the altitude of the aircraft. For example, ambient 
temperature variations can cause the water contaminants in the fuel to condense and settle at the bottom of the fuel tanks. Later ice crystals may form blocking the filter which interrupts the flow of fuel to engines. But the unique characteristics of ANNs can learn such data variations with the inbuilt rules for the given system. ANNs also maintain long-term memory and distinguish patterns even in changing environments, changing altitudes and noisy surroundings.

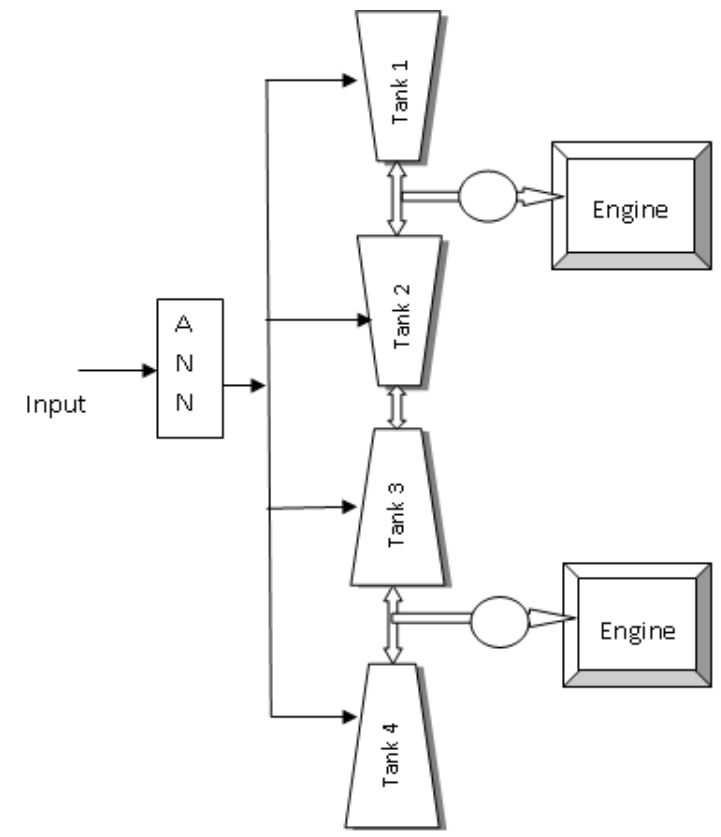

Figure 2. Block diagram of the prognosis tool for a typical aircraft fuel system

Because of these changing features of the fuel system of the aeroplane, ANNs are promising methods for prognostics [14]. This prognosis tool is used to manage and monitor the fuel system and to control the fuel flow as per the fuel consumption rate of the engines. It performs fault detection and the corresponding predictions and suggestions are made so as to maintain constant and required fuel flow rate to the engine throughout the flight.

In this paper, a fuel flow rate prediction model is projected using multilayer Feed-Forward Neural Networks (FFNN). The input-output relation of the FFNN model with two inputs and a single output is as shown in Figure 3.

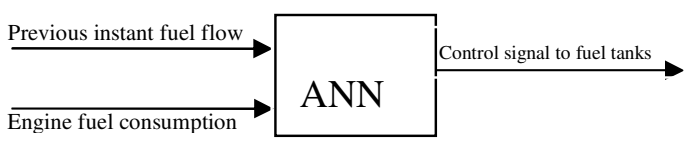

Figure 3. I/P - O/P relation of ANN Model

Back Propagation is an effective training algorithm to minimize the output error. During the process of operation of the fuel system, the BP algorithm measures and calculates the gradient of the error and adjusts the weights of the neural model with respect to the required fuel flow rate. Thus, the ANN prediction model generates the necessary control signals to fetch the required fuel flow rate to the engine. Figure 4 shows an approach for updating process of the ANN model. For maintenance of fuel system, the maintenance engineers generally follow a scheduled maintenance regime. Timely maintenance keeps the working condition of the fuel system within the required 
range of operation. Any leakage in tanks, pumps failure or other faults can alter the operation or may lead to damage of aircraft. Therefore, it is necessary to continuously update the ANN model with the current data, to maintain the required fuel flow rate.

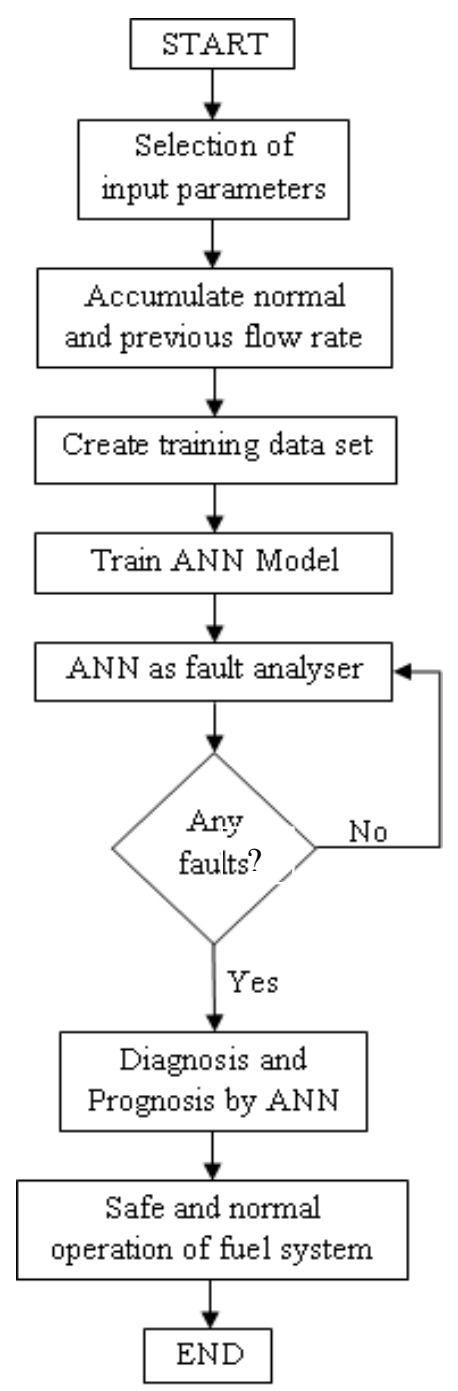

Figure 4. Flowchart of ANN based prognostic tool for fuel system

A Fuel Management System (FMS) gives fuel measurements based on distance to travel, wind and time. When an aeroplane is programmed for a flight route, the fuel monitoring and management system have a capability of displaying the total flight endurance, amount of fuel available and an estimation of remaining fuel. The fuel display in the cockpit can be unreliable if there are tank leaks, pipeline leaks, components failure or plumbing malfunctions [15]. The main task of the fuel management system is to provide the estimation of fuel for the complete flight. This estimation in the FMS is obtained by actual rate of fuel consumption and amount of fuel available in the fuel tanks. In the current FMS, maintenance cost is high and need to check the proper functioning of all subsystems to maintain actual fuel flow rate. Any anomaly in the process leads to the catastrophic damage to the system.

Some of the general factors faced during the process of fuel management are fuel exhaustion, fuel starvation, and fuel contamination. Fuel starvation is an onboard condition wherein the engines 
will not receive any information regarding the availability of fuel. Fuel exhaustion is another condition where the aeroplane's engines are running out of fuel because of some malfunction in the fuel system. Presence of foreign particles like water, surfactants, dirt in the fuel cause fuel contamination which may lead to engine breakdown through damaging or the blocking of the fuel system subcomponents [16]. Hence, this ANN prognosis tool helps to detect and diagnose the occurrence of any kind of faults, which is not possible with the programmed fuel management system. Also, with the proposed tool, redundant components in the fuel system can be reduced.

\section{SIMULATION RESULTS AND DISCUSSION}

The ANN-based prognostic tool is implemented in MATLAB/Simulink. In this work, the model of the aircraft fuel system is simulated similar to the methodology of the paper [17]. The fuel management process is visualized using the ANN-based prognostic tool. The simulated model of a typical aircraft fuel system is as shown in Figure 5. Simulink model of the fuel tank, fuel pump, fuel line and geometry of the aircraft fuel tank are simulated and details of the same are available in the paper [18]. For simulation, the fuel assumed is the liquid Hyjet-4A of which the characteristics are available in the simulink toolbox. The fuel temperature of $22.72^{\circ} \mathrm{C}$ and the viscosity of 1 are assumed respectively. The Simulink model of aircraft fuel pipeline with an internal diameter of $10 \mathrm{~mm}$ geometry factor of 64 is built similar to the actual pipelines with metal pipes. An axial-centrifugal pump with electric driven motor is modeled and opted in the place of the actual fuel pump. During simulation of the fuel pump, the angular velocity of $1770 \mathrm{rpm}$ and the correction factor of 0.8 are set.

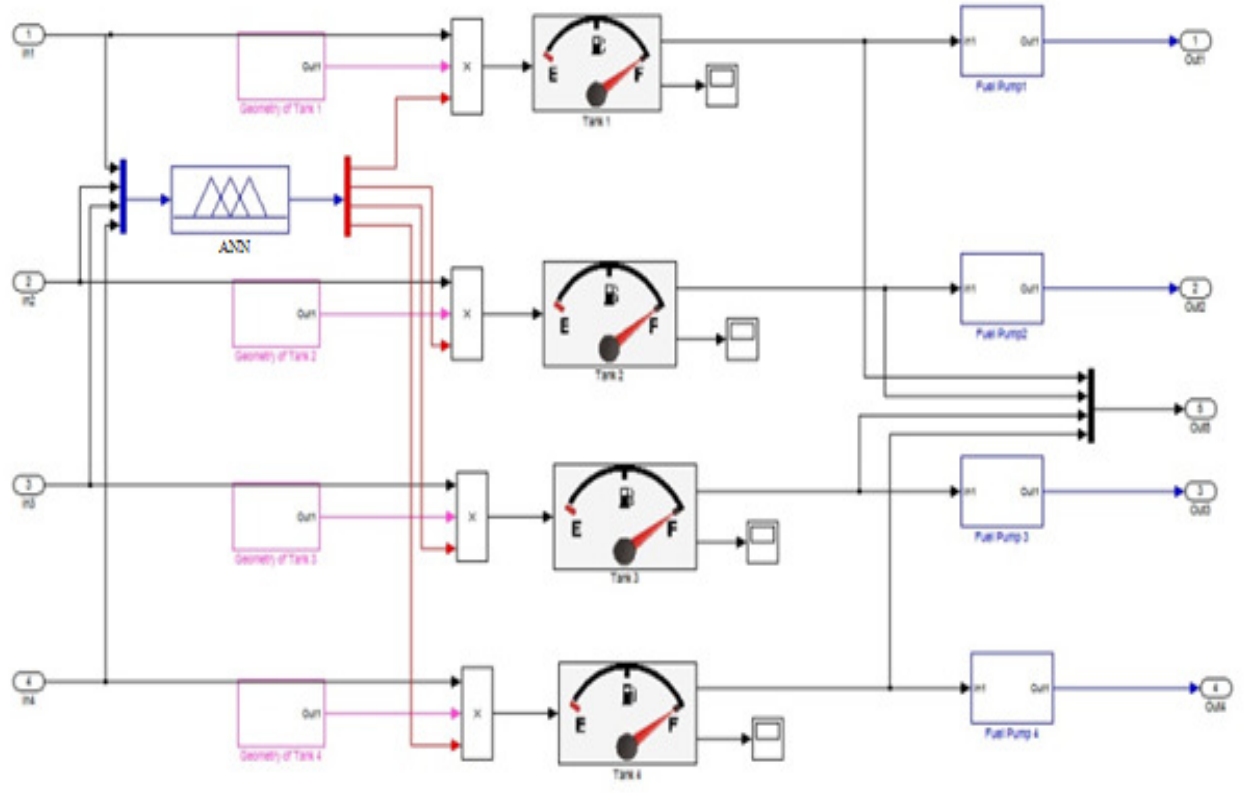

Figure 5. The Simulink model of the aircraft fuel system with ANN as a controller

The fuel system is exposed to inertia, vibration, fluid, and load of aircraft during operation which has to be considered without breakdown. The content of fuel tank(s) should provide at least 30 minutes of continuous engine operation with full power. The Simulink model of a simple four tank fuel system is designed along with fuel pumps, pipelines, and fuel indications. As a controller, the ANN-based prognostic engine is connected. It detects the fault occurrences and takes necessary action to correct by training the neurons according to the input parameters. The output generated from the ANN model are the control signals obtained based on the previous instant flow rate of fuel and rate of fuel consumed by the engines. Thus, the control signal fetches 
the required rate of fuel to the engine(s) without any change, irrespective of any anomalies during operation. It takes a few minutes and/or hours to visualize the fuel leaks because usually, fuel has a slow evaporation rate.

Hence, it becomes difficult to identify fuel leaks immediately. The effectiveness of this method is evaluated by the recognized results with the proposed ANN prognostic technique. Twenty seconds of simulation time is used in this model. The fuel management test result without a controller is depicted in Figure 6a and the fuel consumption requirement is illustrated in Figure $6 b$.

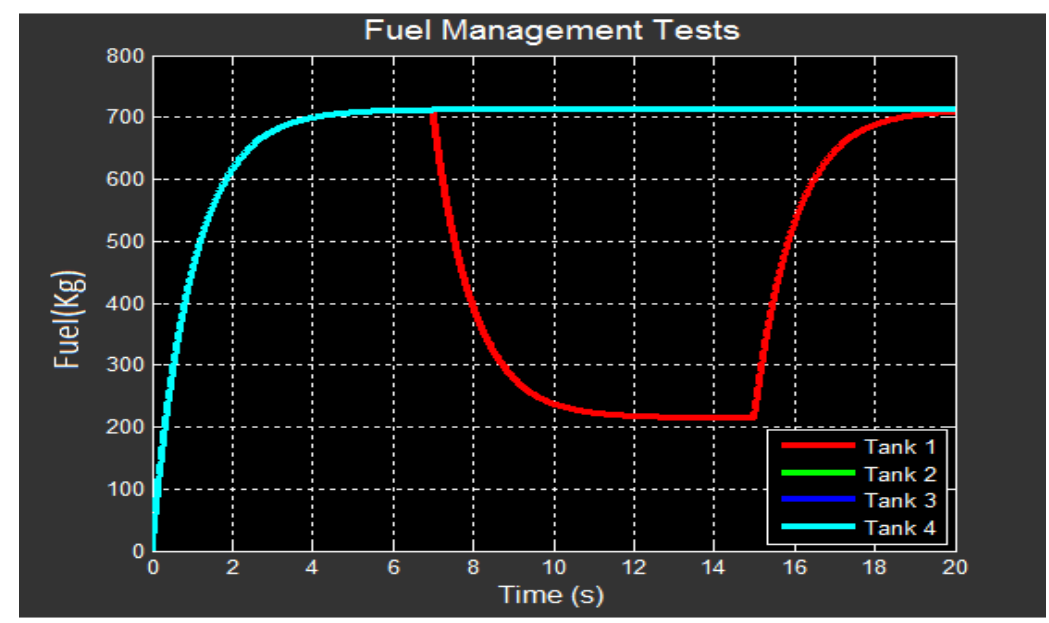

Figure 6a. Fuel management in the aircraft fuel system without a controller

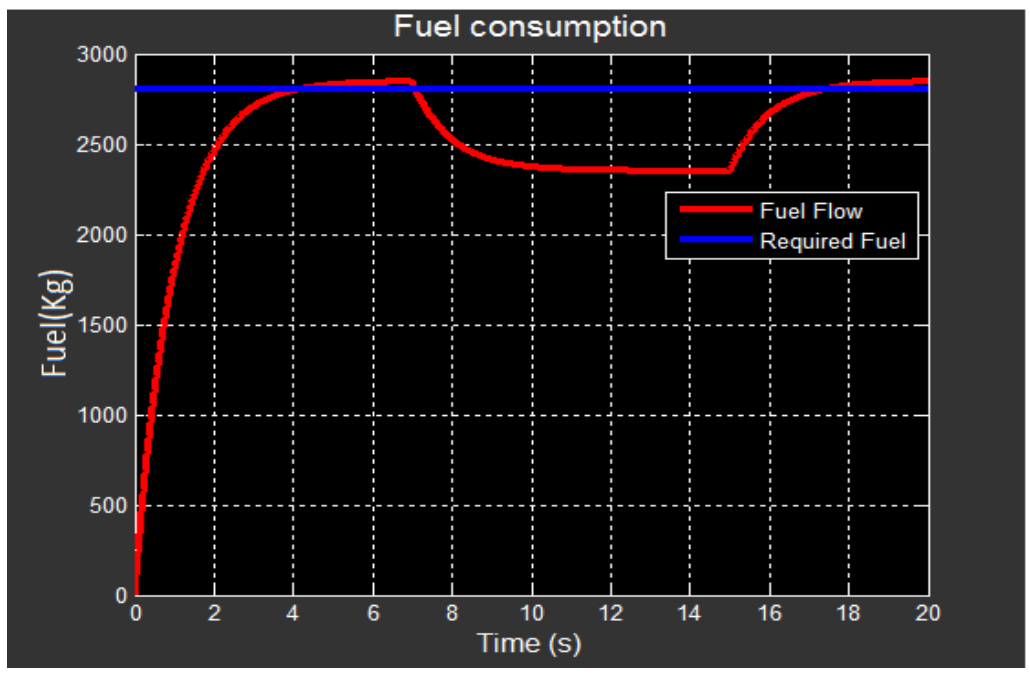

Figure $6 \mathrm{~b}$. Fuel consumption in the engine of the aircraft fuel system without a controller

From Figure $6 \mathrm{~b}$ it is clear that the required fuel for a small aircraft fuel system considered is about $2800 \mathrm{~kg} / \mathrm{hr}$, which is fulfilled by four fuel tanks with each of $700 \mathrm{~kg} / \mathrm{hr}$ within 4 to 6.5 seconds. After 4 seconds, the level of fuel in one of the tanks is reduced due to the faults. During the simulation, the fuel level is reduced intentionally by changing the inputs of ANN accordingly. Thus, the delivery of the fuel and fuel flow rate to the engine is affected. This sudden decrease of fuel (fault) is not correctly identified by the automatic or programmed fuel management system. 
Therefore, the performance of the fuel system gets affected by the change in the rate of fuel flow. Figure 7 shows the fuel management tests using the ANN controller as prognosis tool, which detects the decrease of fuel level in tank 1 and diagnoses it, by fetching the required rate of fuel from other tanks. Thus, this technique helps to maintain the fuel flow rate and avoid unnecessary landing of aircraft or any other kind of critical situations.

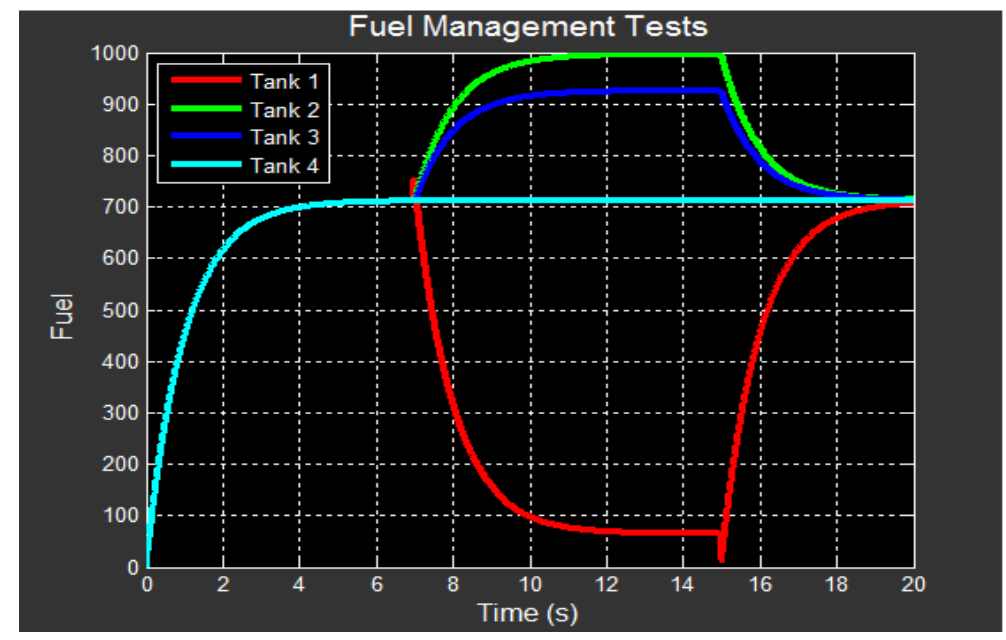

Figure 7. Fuel management in the aircraft fuel system using ANN

The approach of ANN technique as a prognostic tool to manage the fuel is more efficient method compared to other programmed fuel management systems. It detects the time of the fault, diagnoses it and also takes the corrective steps to mitigate it, by fetching the required fuel from the other remaining tanks. The weight updation process of the BP algorithm used by the ANN technique identifies the occurrence of faults and corrects to maintain the required fuel rate. This tool can manage fuel flow of $2600 \mathrm{~kg} / \mathrm{hr}$ as shown in Figure 8.

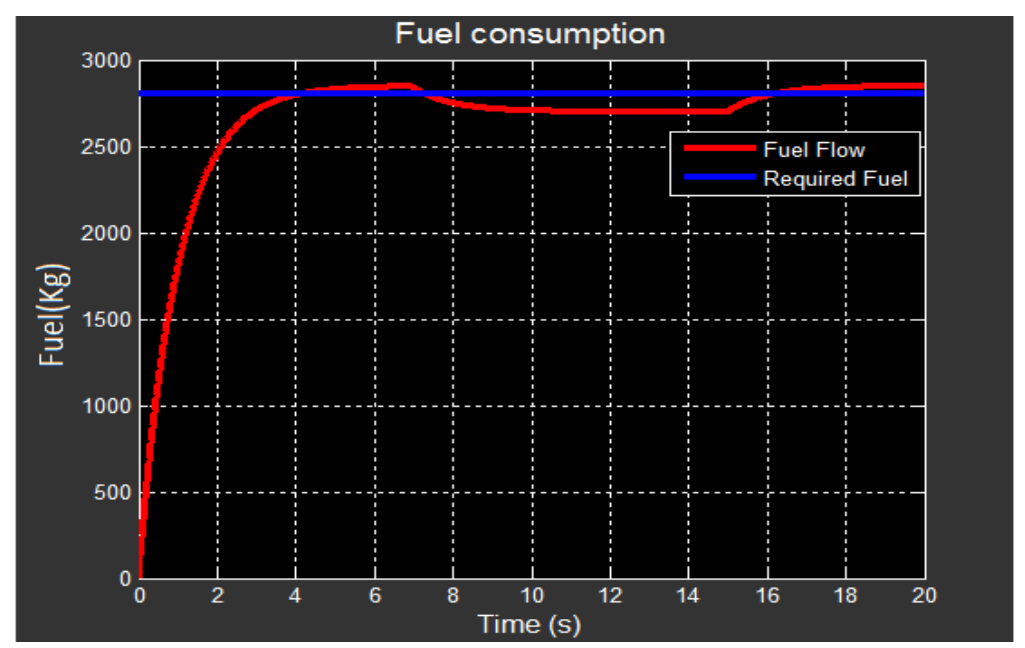

Figure 8. Fuel consumption in the engine of the aircraft fuel system using ANN 


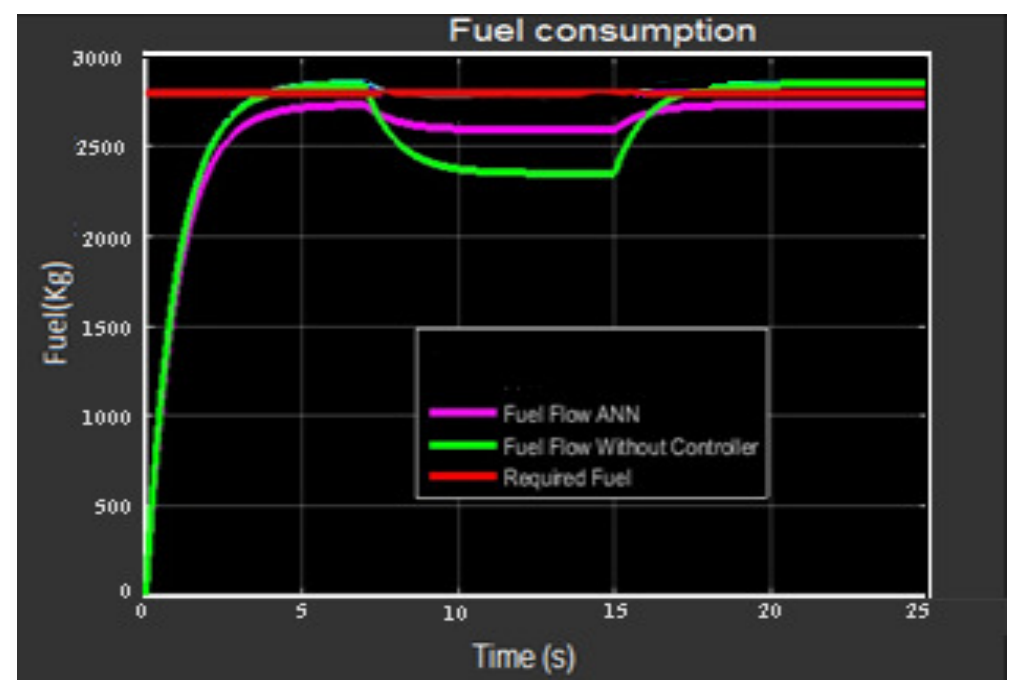

Figure 9. Comparison of fuel consumption

From the comparison result, as shown in Figure 9, the proposed method effectively detects the fault in the fuel tank and manages the fuel requirement of the aircraft engine, with management tests performed without any controller.

\section{CONCLUSION}

Prognostics is a process of failure analysis followed by the health prediction of the system. We have developed an artificial neural network-based fault prognosis tool for a typical four tank aircraft fuel subsystem in this paper. This method using ANN promises to deliver and manage the fuel flow and helps to monitor the fuel level in each tank of the fuel system. The proposed prognosis tool identifies the presence of faults, mitigates them and maintains the proper fuel flow to the engine at the required fuel consumption rate by generating the proper output signal. The efficiency of the simulated model is verified through a comparison with the same fuel system without a controller. From the comparison analysis, it is shown that this prognostic tool employs a unique and effective methodlogy to detect, diagnose and mitigate the fault conditions. The tool is simulated in MATLAB and Simulink for a laboratory environment.

\section{REFERENCES}

[1] Serdar Uckun, Kai Goebel, and Peter J.F. Lucas, "Standardizing research methods for prognostics", 2008 International Conference on Prognostics and Health Management.

[2] Michael Pecht, "Prognostics and health management of Electronics", Wiley 2008G.

[3] Biswas Gautam, Gyula Simon, Nagabhushan Mahadevan, Sriram Narasimhan, John Ramirez and Gabor Karsai, "A robust method for hybrid diagnosis of complex systems", in Proceedings of the 5th Symposium on Fault Detection, Supervision and Safety for Technical Processes, pp.1125-1131, 2003.

[4] Inseok Hwang, Sungwan Kim, Youdan Kim and Chze Eng Seah, "A survey of fault detection, isolation, and reconfiguration methods", IEEE Transactions on Control Systems Technology, Vol.18, No.3, pp.636-653, 2010.

[5] Nikhil M. Vichare and Michael Pecht, "Prognostics and health management of electronics", IEEE Transactions on Components and Packaging Technologies, Vol 29, No. 1, March 2006. 
[6] Isermann Rolf and Peter Balle, "Trends in the application of model-based fault detection and diagnosis of technical processes", Control engineering practice, Vol.5, No.5, pp.709-719, 1997.

[7] R. Isermann, "Supervision, fault-detection and fault diagnosis methods - an introduction", Control Engineering Practice, Vol. 5, No. 5, pp. 639-652, (1997).

[8] Talebi H A and K Khorasani, "A neural network-based multiplicative actuator fault detection and isolation of nonlinear systems", IEEE Transactions on Control Systems Technology, Vol.21, No.3, pp.842-851, 2013.

[9] Tayarani-Bathaie Seyed Sina, Zakieh Nasim Sadough Vanini and Khashayar Khorasani, "Dynamic neural network-based fault diagnosis of gas turbine engines", Neurocomputing, Vol.125, No.11, pp.153-165, 2014.

[10] Zhang Xiaodong, Thomas Parisini and Marios M Polycarpou, "Sensor bias fault isolation in a class of nonlinear systems", IEEE Transactions on Automatic Control, Vol.50, No.3, pp.370-376, 2005.

[11] S. S. Haykin, Neural networks and learning machines, 3. ed. Upper Saddle River: Pearson Education, 2009.

[12] Shen Ting, Fangyi Wan, Weimin Cui, and Bifeng Song, "Application of prognostic and health management technology on aircraft fuel system", In Prognostics and Health Management Conference of IEEE, pp.1-7, 2010.

[13] Jimenez Juan F, Jose M Giron-Sierra, C Insaurralde and M Seminario, “A simulation of aircraft fuel management system”, Simulation Modelling Practice and Theory, Vol.15, No.5, pp.544-564, 2007.

[14] M. Yu, D. Wang, M. Luo, and L. Huang, "Prognosis of hybrid systems with multiple incipient faults: Augmented global analytical redundancy relations approach," IEEE Trans. Syst., Man, Cybern. A Syst., Humans, vol. 41, no. 3, pp. 540-551, May 2011.

[15] www.flightlearnings.com/2017/08/02/fuel-management-systems Date of access:18/6/18.

[16] “Aircraft fuel system" chapter 14 published by Federal Aviation Administration (FAA).

[17] Robert Breda, Vladimir Beno, "Modeling of the control circuit of aircraft fuel system", Przegląd Elektrotechniczny, Vol.89, pp.172-175, 2013.

[18] Vijaylakshmi Jigajinni, Upendranath Vanam, "ANFIS based fault diagnosis tool for a typical small aircraft fuel system" Part of the Advances in Intelligent Systems and Computing book series (AISC, volume 479) ISBN: 9789811017087 (online) 9789811017070 (print) DOI: 10.1007/978-981-101708-745.

\section{AUTHORS}

Mrs. Vijaylakshmi S. Jigajinni obtained Bachelor's degree in Instrumentation Technology from Visveshvaraya Technological University (VTU) of Belgaum590018, Karnataka, India in the year 2003 and Master's degree in Digital Communication from the same university during 2009. Currently, she is an Assistant Professor at Department of Electronics and Communication Engineering of Basaveshwar Engineering (Autonomous) College, Bagalkot, affiliated to VTU, Belagavi, Karnataka, India. Her areas of interests include Artificial Intelligence, Sensors, and Control systems.

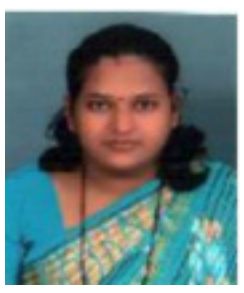


Dr. Vanam Upendranath obtained his Master's degree in Electronics from REC/NIT Warangal, India in 1981, and Ph.D. from University of Trento, Italy in 2005. He was a Scientist in Electronics Systems Area at Central Electronics Engineering Research Institute (CSIR-CEERI), Pilani during 1983- 2010. He was also a Visiting Researcher at the ECE Dept., Johns Hopkins University, the USA during his Ph.D. tenure. From 2010 onwards he has been associated with Integrated Vehicle Health Management (IVHM) program at National Aerospace Laboratories (CSIR-NAL), Bangalore. His areas of interest include Embedded Systems, Wireless Sensor Networks and IVHM for aerospace applications.

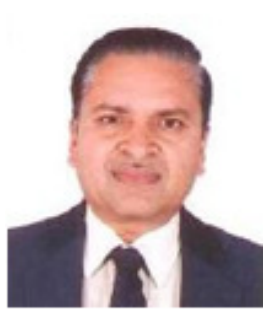

